\title{
The nature of bilateral symmetry
}

Erokhin I.L.

"NBK" LLC, Moscow, Russia

e-mail:i.erokhin@inbox.ru

According to the Genome Tree Theory, in terms of graph theory, the genome of a multicellular organism is structured in the form of an oriented binary tree, vertices of which correspond to the same type of logical elements "genome tree loop step", and the arcs transfer the control between the "steps". The step of the Genome Tree may be represented by three of non-coding regulatory genes with identical complex promotors. Each cell of a multicellular organism has its own step in its Genome Tree. It receives control from the step of the mother cell, initiates the execution of the "cell program" that determines its development and multiplication, regains control after it is completed, and transfers the control to the steps of the daughter cells. The classic description of the cell cycle leaves behind such important issues as the change in the orientation of the mitotic spindle of cell division, the "assembly" of membrane organelles and their spatial arrangement in the daughter cells taking into account the new cell orientation. Orientation of the mitotic spindle over any direction can be submitted in a spherical coordinate system by zenith and azimuthal angles. Cell cycle events are determined by the cell program, which is a multi-level nested Dijkstra loop organized mostly on noncoding regulatory genes. The cell program can be represented by one of the standard cell programs and some combination of subroutines. The change in the zenith and azimuth angles between the spindle apparatus of the mother cell and that of the daughter cell is presumably set by one of such subroutines. The progenitor cell of a symmetric organ or a group of cells divides into two daughter cells. The main feature of the bilateral symmetry of multicellular organisms (including plants) is presumably the use of the same branch of the Genome Tree by these two daughter cells in their subsequent cell cycles. Asymmetric organs and groups of cells of an organism develop from other cells under the control of other branches of the Genome Tree. 